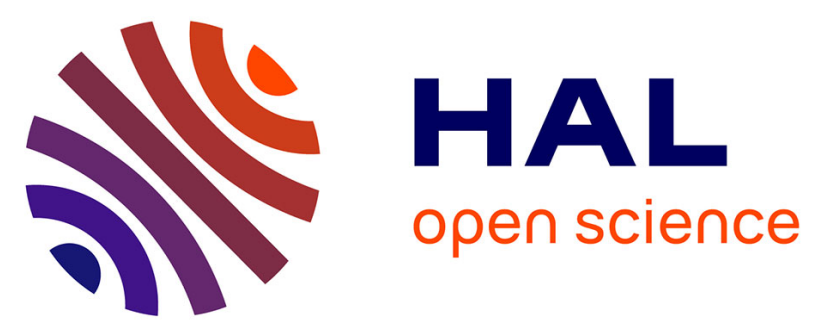

\title{
A Tensor Decomposition Approach to Noninvasive Atrial Activity Extraction in Atrial Fibrillation ECG
}

Lucas Nogueira Ribeiro, Antonio Hidalgo-Muñoz, Gérard Favier, João César

Moura Mota, André L. F. de Almeida, Vicente Zarzoso

\section{- To cite this version:}

Lucas Nogueira Ribeiro, Antonio Hidalgo-Muñoz, Gérard Favier, João César Moura Mota, André L. F. de Almeida, et al.. A Tensor Decomposition Approach to Noninvasive Atrial Activity Extraction in Atrial Fibrillation ECG. 23rd European Signal Processing Conference (EUSIPCO-2015), Aug 2015, Nice, France. pp.2576-2580, 10.1109/EUSIPCO.2015.7362850 . hal-01302686

\section{HAL Id: hal-01302686 \\ https://hal.science/hal-01302686}

Submitted on 14 Apr 2016

HAL is a multi-disciplinary open access archive for the deposit and dissemination of scientific research documents, whether they are published or not. The documents may come from teaching and research institutions in France or abroad, or from public or private research centers.
L'archive ouverte pluridisciplinaire HAL, est destinée au dépôt et à la diffusion de documents scientifiques de niveau recherche, publiés ou non, émanant des établissements d'enseignement et de recherche français ou étrangers, des laboratoires publics ou privés. 


\title{
A TENSOR DECOMPOSITION APPROACH TO NONINVASIVE ATRIAL ACTIVITY EXTRACTION IN ATRIAL FIBRILLATION ECG
}

\author{
L. N. Ribeiro ${ }^{* \dagger}$, A. R. Hidalgo-Muñoz ${ }^{*}$, G. Favier ${ }^{*}$, J. C. M. Mota ${ }^{\dagger}$, A. L. F. de Almeida ${ }^{\dagger}$ and V. Zarzoso* \\ * I3S Laboratory, University of Nice Sophia Antipolis, CNRS, France \\ ${ }^{\dagger}$ GPSI Laboratory, Federal University of Ceará, Fortaleza, Brazil
}

\begin{abstract}
Atrial fibrillation $(A F)$, the most common arrhythmia in adults, is still considered as the last great frontier of cardiac electrophysiology, since its mechanisms are not completely understood. Analysis of the atrial activity (AA) signal contained in electrocardiograms during AF episodes is a noninvasive and inexpensive solution for obtaining useful information about AF. This work presents tensor decompositions as an alternative to classic blind source separation methods based on matrix decompositions due to their appealing uniqueness properties and considers in particular the block term decomposition (BTD). The practical usefulness of BTD is evaluated by comparing its AA estimation quality, measured by spectral concentration, to those of two benchmark methods, revealing that BTD presents a better performance. The results presented in this work motivate further investigation of tensor decompositions for AF analysis.
\end{abstract}

Index Terms - Atrial fibrillation, blind source separation, tensor decompositions, electrocardiogram

\section{INTRODUCTION}

Atrial fibrillation (AF) is the most common sustained cardiac arrhythmia in adults. It consists in apparently disorganized impulses typically generated around the pulmonary veins, leading to rapid $\mathrm{f}$-waves of atrial activity (AA) instead of the $\mathrm{P}$ wave preceding the QRS complex in the electrocardiogram (ECG). Currently, AF is still considered as the last great frontier of cardiac electrophysiology, creating a significant interest in diverse disciplines of knowledge including applied mathematics and signal processing.

A good AA estimation is the first step needed to achieve a rigorous analysis of AF. Classical AA estimation methods [1] are based on averaging the ventricular activity (VA) and then subtracting the obtained template from QT segments. However, since this approach relies on beat detection, it is very sensitive to ectopic beats. Also, by construction, this approach cancels out only the VA, ignoring other interference

L. N. Ribeiro is supported by CNPq/CAPES (Brazil). A.R. HidalgoMuñoz is supported by a Postdoctoral Research Fellowship awarded by the University of Nice Sophia Antipolis. V. Zarzoso is a member of the Institut Universitaire de France. sources. In order to surmount these shortcomings, there has been an interest in blind source separation (BSS) techniques. Basically, techniques such as principal component analysis (PCA) and independent component analysis (ICA) have been used to estimate the AA from ECG recordings [2-4].

The BSS methods mentioned above are based on matrix decompositions, which require strong mathematical constraints over its factors to assure the decomposition uniqueness and perform the source separation. For instance, constraints such as statistical independence between the signal components and mutual orthogonality on the factor columns are frequently imposed. Moreover, PCA and ICA are statistical approaches, implying that the AA estimation quality depends on the sample size. The impossibility of dealing with the underdetermined scenario, i.e., when there are more sources than leads (limited spatial diversity), is a serious drawback of matrix-based BSS methods. To solve these limitations, tensor decompositions have been presented as a promising tool [5]. In short, a higher-order tensor ( $P$ th order, $P>2$ ) can be seen as the generalization of usual matrices to multidimensional arrays. An attractive property of high-order tensors is the fact that they can be uniquely decomposed provided that mild conditions be fulfilled [5], allowing the development of more robust BSS methods. The present work explores the application of higher-order tensor decompositions to noninvasive AA estimation.

Tensor decompositions have been exhaustively reviewed in the recent literature, e.g., [5,6] among other works. They have already been employed in biomedical engineering problems including space-time-frequency analysis of EEG data during an epileptic seizure [7] and EEG source localization [8]. Regarding ECG processing, higher-order tensors have been used for feature extraction and classification of ECG [9] and fetal ECG extraction [10]. In [11], the block term decomposition (BTD) was introduced as a deterministic BSS method that supposes that the underlying sources can be modeled as a sum of complex exponentials. In fact, this is a plausible assumption for biomedical signals, inspiring the application of this tensor decomposition on EEG analysis during epileptic seizures [12]. BTD was shown to be a powerful BSS method when its parameters are well chosen. Nevertheless, an automatic parameter selection method for 
BTD remains an open problem.

In this paper, we propose BTD as a new AA extraction approach that overcomes the limitations of matrix methods in temporally and spatially constrained scenarios. To this aim, it is supposed that the f-waves can be represented as a sum of complex exponentials. To assure a satisfactory extraction quality, it is necessary to select good parameters for the decomposition. An established atrial signal model during AF will be analyzed to provide some guidelines for parameter selection. Subsequently, the usefulness of BTD will be evaluated on a real AF ECG by comparing its performance to those of two AA extraction methods based on matrix decompositions: RobustICA-f [4] and PCA [13].

\section{NONINVASIVE AA EXTRACTION IN AF ECGS}

\subsection{Matrix decomposition approach}

BSS approaches based on linear instantaneous mixtures have been widely used to model the AA extraction problem [2-4]. These methods aim to extract the atrial activity from multilead ECG based on the model $\mathbf{Y}=\mathbf{M S}$, where $\mathbf{Y} \in \mathbb{R}^{K \times N}$ is the ECG data matrix with $K$ leads and $N$ samples, $\mathbf{M} \in \mathbb{R}^{K \times R}$ is the mixing matrix and $\mathbf{S} \in \mathbb{R}^{R \times N}$ is the source matrix with $R$ sources. The coefficients of $\mathbf{M}$ reflect the propagation characteristics of the body and $\mathbf{S}$ contains the ventricular, atrial and noise sources [2]. BSS methods seek to recover $\mathbf{S}$ and possibly $\mathbf{M}$ knowing only $\mathbf{Y}$. In fact, this inverse problem presents infinite solutions, and it is then necessary to reduce the possible solutions using a priori knowledge on the sources and/or the mixing system. During AF, atrial and ventricular sources can be considered to be statistically independent or at least uncorrelated, since atrial wavefronts lead to ventricular depolarization at irregular instants [2]. It can also be assumed that the QRST complex can be linked to super-Gaussian components, while the f-wave presents a near-Gaussian probability density function. However, it is known that AA is a narrowband signal. Therefore, transforming it into the frequency domain leads to a super-Gaussian component, property exploited by RobustICA-f [4]. The narrowband character of the AA could be further exploited to support the signal model based on a sum of complex exponentials implicitly assumed by the tensor technique presented next.

\subsection{Tensor approach via block term decomposition}

Consider a third-order tensor $\mathcal{T} \in \mathbb{R}^{I_{1} \times I_{2} \times I_{3}}$. Its BTD is defined as

$$
\mathcal{T}=\sum_{r=1}^{R} \mathbf{E}_{r} \circ \mathbf{c}_{r}
$$

where "o" denotes the outer product, $\mathbf{E}_{r} \in \mathbb{R}^{I_{1} \times I_{2}}$ has rank $L_{r}$ and $\mathbf{c}_{r} \in \mathbb{R}^{I_{3}}$. If $\mathbf{E}_{r}$ admits a decomposition $\mathbf{E}_{r}=$ $\mathbf{D}_{r}^{(1)} \mathbf{D}_{r}^{(2)^{T}}$, where $\mathbf{D}_{r}^{(1)} \in \mathbb{R}^{I_{1} \times L_{r}}$ and $\mathbf{D}_{r}^{(2)} \in \mathbb{R}^{I_{2} \times L_{r}}$ have rank $L_{r}$, then (1) can be expressed as

$$
\mathcal{T}=\sum_{r=1}^{R}\left(\mathbf{D}_{r}^{(1)} \mathbf{D}_{r}^{(2)^{T}}\right) \circ \mathbf{c}_{r}
$$

Note that each $r$ th term of (2) has multilinear rank $\left(L_{r}, L_{r}, 1\right)$. This decomposition will be unique up to permutation and scaling provided that the factors $\mathbf{D}^{(1)}=\left[\mathbf{D}_{1}^{(1)}, \mathbf{D}_{2}^{(1)}, \ldots\right.$, $\left.\mathbf{D}_{R}^{(1)}\right]$ and $\mathbf{D}^{(2)}=\left[\mathbf{D}_{1}^{(2)}, \mathbf{D}_{2}^{(2)}, \ldots, \mathbf{D}_{R}^{(2)}\right]$ are full-column rank with $I_{1}, I_{2} \geq \sum_{r=1}^{R} L_{r}$ and $\mathbf{C}=\left[\mathbf{c}_{1}, \mathbf{c}_{2}, \ldots, \mathbf{c}_{R}\right]$ does not contain collinear columns [11].

In order to obtain a third-order tensor from $\mathbf{Y}$, each $k$ th row of this matrix is mapped onto a $(I \times J)$ Hankel matrix, with $I+J-1=N$, where $I=J=\frac{N+1}{2}$ if $N$ is odd, or $I=$ $\frac{N}{2}$ and $J=\frac{N}{2}+1$ if $N$ is even. Then each Hankel matrix is stacked along the third dimension of a tensor $\mathcal{Y} \in \mathbb{R}^{I \times J \times K}$, which can be defined as

$$
[\mathcal{Y}]_{i, j, k}=\sum_{r=1}^{R} m_{k, r} s_{r, i+j-1}
$$

in which $m_{k, r}$ and $s_{r, i+j-1}$ are the $(k, r)$ and $(r, i+j-1)$ entries of $\mathbf{M}$ and $\mathbf{S}$, respectively, for $1 \leq i \leq I, 1 \leq j \leq$ $J, 1 \leq k \leq K$. The $k$ th matrix slice of $\mathcal{Y}$ can be expressed as

$$
[\mathcal{Y}]_{:, i, k}=\sum_{r=1}^{R} m_{k, r} \mathbf{H}_{\mathbf{S}}^{(r)}
$$

where $\mathbf{H}_{\mathbf{S}}^{(r)} \in \mathbb{R}^{I \times J}$ is a Hankel matrix obtained from the $r$ th row of $\mathbf{S}$. In equation (3), for a fixed $r$ and $k=1, \ldots, K$, the matrix $\mathbf{H}_{\mathbf{S}}^{(r)}$ is scaled by $m_{k, r}$. This implies that for a fixed $r$, the outer product between $\mathbf{H}_{\mathbf{S}}^{(r)}$ and $\mathbf{m}_{r}$, the $r$ th column of $\mathbf{M}$, is being performed to yield their contribution to $\mathcal{Y}$. Now varying $k$, we can write in tensor form:

$$
\mathcal{Y}=\sum_{r=1}^{R} \mathbf{H}_{\mathbf{S}}^{(r)} \circ \mathbf{m}_{r}
$$

which is equivalent to the BTD model (1). Now, suppose that the $r$ th ECG source can be written as:

$$
s_{r, n}=\sum_{l_{r}=1}^{L_{r}} \lambda_{l_{r}, r} z_{l_{r}, r}^{n-1}, \quad 1 \leq n \leq N, 1 \leq r \leq R
$$

where $L_{r}$ is the number of exponential terms, $\lambda_{l_{r}, r}$ is the linear coefficient and $z_{l_{r}, r}$ is the base of the exponential associated with the $l_{r}$ th term of $s_{r, n}$, also called pole in [11]. Since the sources are modeled by (5), their associated Hankel matrix admits the following Vandermonde decomposition [14]:

$$
\mathbf{H}_{\mathbf{S}}^{(r)}=\mathbf{V}_{r} \operatorname{diag}\left(\lambda_{1, r}, \lambda_{2, r}, \ldots, \lambda_{L_{r}, r}\right) \hat{\mathbf{V}}_{r}^{T}
$$


where the Vandermonde matrices $\mathbf{V}_{r} \in \mathbb{R}^{I \times L_{r}}$ and $\hat{\mathbf{V}}_{r} \in$ $\mathbb{R}^{J \times L_{r}}$ are, respectively,

$\mathbf{V}_{r}=\left[\begin{array}{cccc}1 & 1 & \ldots & 1 \\ z_{1, r} & z_{2, r} & \ldots & z_{L_{r}, r} \\ \vdots & \vdots & & \vdots \\ z_{1, r}^{I-1} & z_{2, r}^{I-1} & \ldots & z_{L_{r}, r}^{I-1}\end{array}\right], \hat{\mathbf{V}}_{r}=\left[\begin{array}{cccc}1 & 1 & \ldots & 1 \\ z_{1, r} & z_{2, r} & \ldots & z_{L_{r}, r} \\ \vdots & \vdots & & \vdots \\ z_{1, r}^{J-1} & z_{2, r}^{J-1} & \ldots & z_{L_{r}, r}^{J-1}\end{array}\right]$.

Since a Vandermonde matrix with $I, J \geq L_{r}$ generated by distinct poles has full-column rank $L_{r}$, model (4) will be unique if all the poles $z_{l_{r}, r}$ are distinct for $1 \leq l_{r} \leq L_{r}, 1 \leq$ $r \leq R$, and $\mathbf{M}$ does not have proportional columns.

In order to calculate the BTD of a higher-order tensor, the number $R$ of block components and the multilinear rank $\left(L_{r}, L_{r}, 1\right), r=1, \ldots, R$, need to be determined. From equation (6), symbol $L_{r}$ denotes the number of poles that generate the $r$ th source. Note that this number is also revealed by $\operatorname{rank}\left(\mathbf{H}_{\mathbf{S}}^{(r)}\right)$.

\subsection{Atrial signal modeling during AF}

The model proposed by Stridh and Sörnmo [15] mimics the sawtooth pattern of that typically characterizes the AA signal during AF, especially in early forms of the disease. It consists of a sinusoid and $(M-1)$ harmonics modulated in amplitude and frequency. Thus the f-wave is modeled by

$$
f(n)=-\sum_{i=1}^{M} a_{i}(n) \sin (i \theta(n))
$$

where $a_{i}(n)=\frac{2}{i \pi}\left[a+\Delta a \sin \left(2 \pi \frac{f_{a}}{F_{s}} n\right)\right]$ is the modulated amplitude, in which $a$ is the sawtooth amplitude, $\Delta a$ the modulation peak amplitude, $f_{a}$ the amplitude modulation frequency and $F_{s}$ the sampling frequency. The phase $\theta(n)=2 \pi \frac{f_{0}}{F_{s}} n+\left(\frac{\Delta f}{f_{f}}\right) \sin \left(2 \pi \frac{f_{f}}{F_{s}} n\right)$ varies sinusoidally around $f_{0}$ with maximum frequency deviation $\Delta f$ and modulation frequency $f_{f}$.

Model (7) does not accept an exact all-pole representation as in (5), but it is a useful model for AF and will be used as a reference signal due to its harmonic structure. The numerical analysis of the next section evaluates to what extent model (7) can be approximated by the complex exponential model assumed by BTD.

\section{EXPERIMENTAL RESULTS}

The experiments conducted using the artificial AF model provide guidelines for parameter selection in BTD under Stridh's AA signal model even though it cannot generally be expressed as a sum of complex exponentials. The synthetic AF model proposed above is analyzed in this section to provide information about the multilinear rank of the block terms. In this work, the randomly initialized BTD factors are calculated using the MATLAB implementation [16] of the nonlinear least-squares (NLS) approach proposed in [17]. After carrying out the decomposition, the rank- $L_{r}$ component $\mathbf{H}_{\mathbf{S}}^{(r)}$ is calculated and the $r$ th source is retrieved by averaging its anti-diagonals, as proposed in [11].

Monte Carlo (MC) simulations are performed to find an optimal value of $R$ (the number of block terms in (4)) with respect to the Normalized Mean Squared Error (NMSE), defined as $\mathbb{E}\left[(\hat{s}-f)^{2}\right] / \mathbb{E}\left[f^{2}\right]$, between the estimated signal $\hat{s}$ yielded by BTD and the generated AF signal $f$ given by equation (7). Then, BTD is performed on real ECGs using the obtained parameters and its results are compared to those of benchmark methods. In this case, the spectral concentration (SC) around the dominant or peak frequency $\left(F_{p}\right)$ is used as performance index [3]. The SC is defined as $\frac{\sum_{0.82 F_{p}}^{1.17 F_{A A}} P_{A}\left(f_{i}\right)}{\sum_{0}^{F_{S} / 2} P_{A A}\left(f_{i}\right)}$, where $P_{A A}$ is the power spectrum of the AA signal, which is estimated as in [3].

\subsection{Extraction of simulated AF signals}

To simplify the search for suitable BTD parameters, we consider $L_{r}=L$ for $r=1, \ldots, R$. Henceforth, only $L$ and $R$ need to be determined. If the actual AA signal was available, $L$ could be found by calculating the rank of its associated Hankel matrix as noted at the end of Sec. 2.2. Unfortunately the signal of interest is unavailable, forcing us to deal with an approximation of the actual AA signal. In this paper, the AA is approximated by the model presented in Section 2.3. The parameters of this synthetic model are chosen to be the "case A" set of parameters [15] $(a=150 \mu \mathrm{V}, \Delta a=50 \mu \mathrm{V}$, $f_{a}=0.08 \mathrm{~Hz}, f_{0}=6 \mathrm{~Hz}, \Delta f=0.2 \mathrm{~Hz}, f_{f}=0.1 \mathrm{~Hz}$, and $M=5$ ), which generates an AF pattern with large amplitude and long cycle length. The sampling rate is $F_{s}=1 \mathrm{kHz}$ throughout all experiments presented in this section. Synthetic AF signals with different sample size are generated and the rank of their associated Hankel matrix is calculated, as illustrated in Fig. 1.

Figure 1 shows that $L$ grows with the length of the synthetic AF as would be expected from the fact that the signal does not accept an all-pole model and may be nonstationary. Consequently, the computational cost for performing the decomposition grows as well. Therefore, a small sample size or performing downsampling would be preferred when using BTD.

Once $L$ has been selected, different values of $R$ can be tested in order to find the optimal number of block terms. The following experiment consists of generating synthetic AF ECGs and extracting the generated f-wave using different values of $R$. To generate ventricular interference, sinus rhythm ECG recordings from the PTB Diagnostic ECG database [18] are used. These recordings are preprocessed by a forwardbackward bandpass type-II Chebyshev IIR filter with cut-off frequencies of $0.5 \mathrm{~Hz}$ and $30 \mathrm{~Hz}$ to remove baseline wander and powerline interference. Next, the beginning and the end 


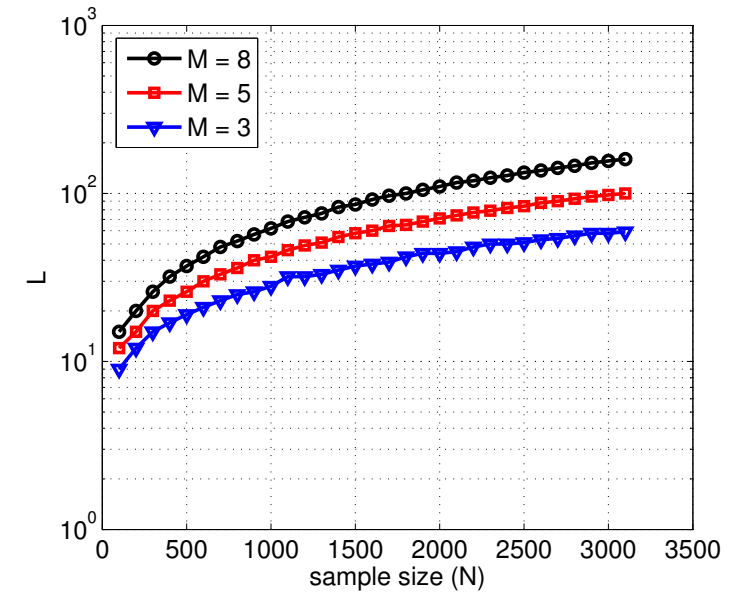

Fig. 1. Relationship between the sample size $N$ and the Hankel matrix rank $L$ for different values of $M$, the number of harmonics in model (7).

of the $\mathrm{P}$ waves associated with each heart beat are manually annotated and each $\mathrm{P}$ wave is suppressed by spline interpolation between its onset and offset points. The resulting multilead signal is stored in matrix $\mathbf{V} \in \mathbb{R}^{K \times N}$, where $K$ is the number of leads and $N$ the number of samples. Then, a synthetic AF signal according to model (7) is generated, and stored in vector $\mathbf{f} \in \mathbb{R}^{N}$. Both ECG leads and f-wave are normalized and subsequently combined in the following manner: $\mathbf{Y}(\sigma)=\mathbf{a f}^{T}+\sigma \mathbf{V}$, where $\mathbf{Y}(\sigma) \in \mathbb{R}^{K \times N}$ is the resulting observed ECG data matrix, $\mathbf{a} \in \mathbb{R}^{K}$ is an unit-length random vector of spatial scales representing the AA source contribution to the ECG leads, and $\sigma$ is the power factor of the VA components. Consequently, the atrio-ventricular power ratio (AVR) can be defined as AVR $[\mathrm{dB}]=-20 \log _{10} \sigma$. Two MC simulations are conducted by varying the sample size and the number of leads and calculating the NMSE for each case, as depicted in Fig. 2, indicating that using either $R=3$ or $R=4$ results in a low estimation error for at least 3 leads and 1000 samples.

\subsection{Real AF ECGs}

Real AF ECG recordings that present an f-wave with high amplitude and long cycle, similar to the synthetic wave used in the previous section, were acquired at a sampling rate of $977 \mathrm{~Hz}$ and preprocessed as in Section 3.1. ${ }^{1}$ To assess the performance of the AA extraction methods in a constrained scenario, only 3 precordial leads (V1-V3) and 1000 samples were considered. The f-wave was estimated by RobustICA- $f$, PCA and BTD, using parameters based on the results obtained with synthetic signals in Sec. 3.1. The rank parameter

\footnotetext{
${ }^{1}$ Dataset kindly provided by the Cardiology Department, Princess Grace Hospital, Monaco.
}
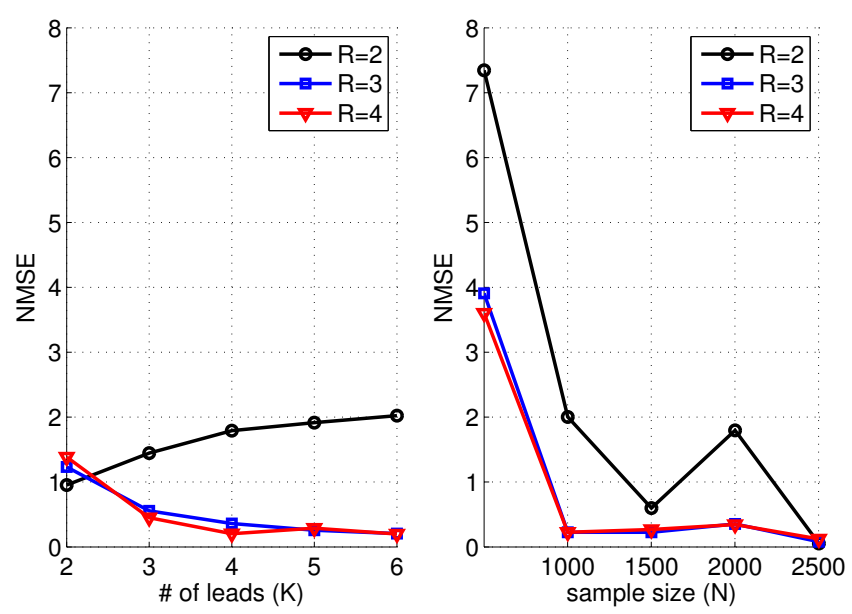

Fig. 2. MC simulations with 50 independent runs evaluating the effect of number of leads $K$ and sample size $N$ on the estimation quality, for AVR $=-6 \mathrm{~dB}$. In the first scenario $N=1000$ samples are used, while $K=6$ leads (V1-V6) are used in the second scenario.

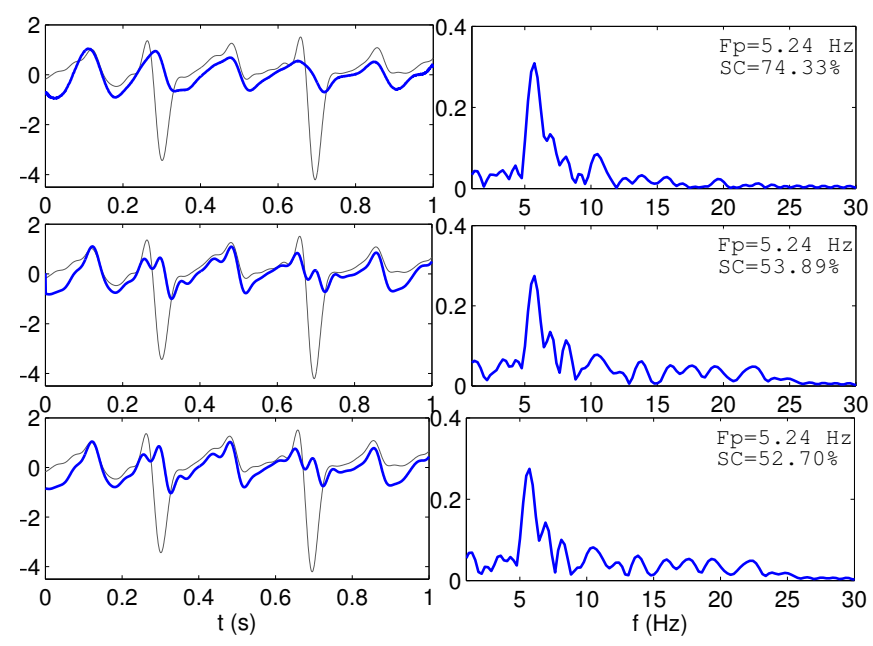

Fig. 3. Estimated AA waveform (left plots) and spectra (right plots) for BTD with $R=3$ and $L=42$ (top), RobustICA-f (middle) and PCA (bottom) with $K=3$ leads and $N=1000$ samples. The vertical axis represents waveform amplitude on the left column and spectral magnitude on the right column. For reference, the V1 lead signal is shown in the background (gray) of the left plots.

$L=42$ was selected by consulting the value corresponding to $N=1000$ samples for $M=5$ harmonics in Fig. 1 and the number of block terms was chosen as $R=3$ according to Fig. 2. The estimated f-waves are shown in Fig. 3. The estimation provided by BTD presents the best SC performance index in this scenario, indicating its superior robustness to the spatial diversity (number of leads) over the matrix methods. 


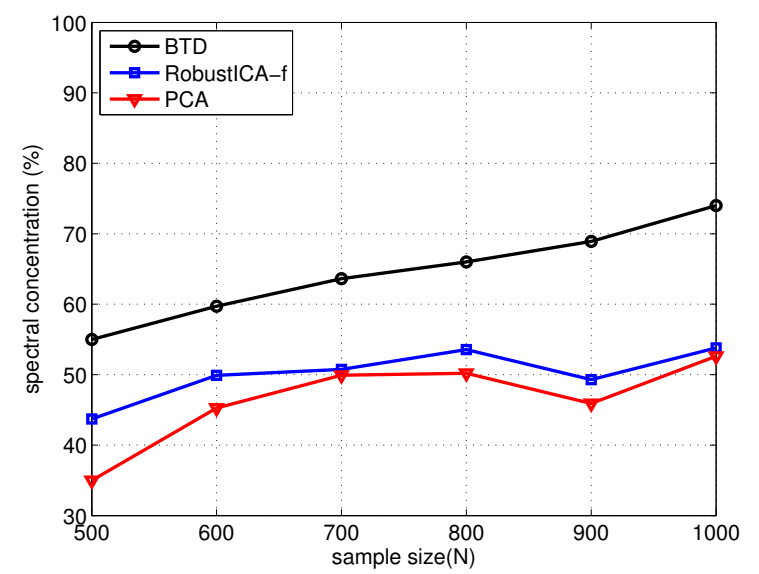

Fig. 4. Spectral concentration vs. sample size $N$ for $K=3$ leads (V1-V3) and $R=3$.

As ground truth, an intracardiac electrogram recorded by a bipolar catheter in the left atrial appendage of the same AF patient presents $F_{p}=5.84 \mathrm{~Hz}$ and $\mathrm{SC}=84.5 \%$.

An additional experiment was carried out by changing the sample size of the ECG recording, estimating the f-wave and calculating its SC, as shown in Fig. 4. The parameter $L$ was computed as in the previous experiment and the number of block terms was $R=3$ as well. These experiments indicate that the f-wave can be approximated as a sum of complex exponentials and that BTD is robust to temporal and spatial constrained scenarios, presenting better SC index over the benchmark methods. It is important to note, however, that a successful tensor decomposition by BTD strongly depends on the proper initialization of its factors. Devising an appropriate initialization for BTD remains a challenge.

\section{CONCLUSIONS}

Tensor decompositions are introduced in this work as a new deterministic method for non-invasive AA extraction intended to overcome the drawbacks of matrix BSS methods. Experiments with real AF ECG signals reveal that BTD can provide a better estimation quality over some classical BSS methods based on matrix decompositions. These results require that suitable parameters be chosen for BTD. In order to provide some guidelines for optimal parameter selection, an established synthetic AF model was used as reference and analyzed by means of numerical simulations, allowing the development of a relationship between the AF model and the BTD parameters. This work was mainly intended as a proof of concept of the applicability of tensor decomposition to noninvasive AA extraction in AF ECGs. Future work could aim at evaluation on a full signal database, integrating other AA a priori information in the BTD approach and considering more realistic AF signal models.

\section{REFERENCES}

[1] L. Mainardi, L. Sornmo, and S. Cerutti, Understanding atrial fibrillation: the signal processing contribution, Morgan \& Claypool Publishers, 2008.

[2] J. J. Rieta, F. Castells, C. Sánchez, et al., "Atrial activity extraction for atrial fibrillation analysis using blind source separation," IEEE Transactions on Biomedical Engineering, vol. 51, no. 7, pp. 11761186, 2004.

[3] F. Castells, J. J. Rieta, J. Millet, et al., "Spatiotemporal blind source separation approach to atrial activity estimation in atrial tachyarrhythmias," IEEE Transactions on Biomedical Engineering, vol. 52, no. 2, pp. 258-267, 2005.

[4] V. Zarzoso and P. Comon, "Robust independent component analysis by iterative maximization of the kurtosis contrast with algebraic optimal step size," IEEE Transactions on Neural Networks, vol. 21, no. 2, pp. 248-261, 2010.

[5] T. G. Kolda and B. W. Bader, "Tensor decompositions and applications," SIAM review, vol. 51, no. 3, pp. 455-500, 2009.

[6] A. Cichocki, D. Mandic, L. De Lathauwer, Guoxu Zhou, Qibin Zhao, C. Caiafa, and H.A. Phan, "Tensor decompositions for signal processing applications: From two-way to multiway component analysis," Signal Processing Magazine, IEEE, vol. 32, no. 2, pp. 145-163, March 2015.

[7] E. Acar, C. Aykut-Bingol, H. Bingol, et al., "Multiway analysis of epilepsy tensors," Bioinformatics, vol. 23, no. 13, pp. 10-18, 2007.

[8] H. Becker, L. Albera, P. Comon, et al., "EEG extended source localization: tensor-based vs. conventional methods," NeuroImage, vol. 96, pp. 143-157, 2014.

[9] K. Huang and L. Zhang, "Cardiology knowledge-free ECG feature extraction using generalized tensor rank one discriminant analysis," EURASIP Journal on Advances in Signal Processing, vol. 2014, no. 1, pp. 1-15, 2014.

[10] M. Niknazar, H. Becker, B. Rivet, et al., "Robust 3-way tensor decomposition and extended state Kalman filtering to extract fetal ECG," in Proceedings of the 21st European Signal Processing Conference (EUSIPCO), Marrakech, Morocco, September 2013.

[11] L. De Lathauwer, "Blind separation of exponential polynomials and the decomposition of a tensor in rank- $\left(L_{r}, L_{r}, 1\right)$ terms," SIAM Journal on Matrix Analysis and Applications, vol. 32, no. 4, pp. 14511474, 2011.

[12] B. Hunyadi, D. Camps, L. Sorber, et al., "Block term decomposition for modelling epileptic seizures," EURASIP Journal on Advances in Signal Processing, vol. 2014, no. 1, pp. 1-19, 2014.

[13] I. Jolliffe, Principal Component Analysis, Wiley Online Library, 2005.

[14] D. L. Boley, F. T. Luk, and D. Vandevoorde, "Vandermonde factorization of a Hankel matrix," in Proceedings of the Workshop on Scientific Computing, Hong Kong, March 1997.

[15] M. Stridh and L. Sörnmo, "Spatiotemporal QRST cancellation techniques for analysis of atrial fibrillation," IEEE Transactions on Biomedical Engineering, vol. 48, no. 1, pp. 105-111, 2001.

[16] L. Sorber, M. Van Barel, and L. De Lathauwer, "Tensorlab v2.0," Available online, January 2014. URL: http://www.tensorlab.net/.

[17] L. Sorber, M. Van Barel, and L. De Lathauwer, “Optimization-based algorithms for tensor decompositions: Canonical polyadic decomposition, decomposition in rank- $\left(L_{r}, L_{r}, 1\right)$ terms, and a new generalization," SIAM Journal on Optimization, vol. 23, no. 2, pp. 695-720, 2013.

[18] A. L. Goldberger, L. A. N. Amaral, L. Glass, et al., "Physiobank, physiotoolkit, and physionet components of a new research resource for complex physiologic signals," Circulation, vol. 101, no. 23, pp. e215-e220, 2000. 